\title{
Phase diagram of Rydberg atoms in a nonequilibrium optical lattice
}

\author{
Jing Qian, Guangjiong Dong, Lu Zhou and Weiping Zhang \\ Quantum Institute for Light and Atoms, Department of Physics, \\ East China Normal University, Shanghai 200062, People's Republic of China
}

\begin{abstract}
We study the quantum nonequilibrium dynamics of ultracold three-level atoms trapped in an optical lattice, which are excited to their Rydberg states via a two-photon excitation with nonnegligible spontaneous emission. Rich quantum phases including uniform phase, antiferromagnetic phase and oscillatory phase are identified. We map out the phase diagram and find these phases can be controlled by adjusting the ratio of intensity of the pump light to the control light, and that of two-photon detuning to the Rydberg interaction strength. When the two-photon detuning is blue-shifted and the latter ratio is less than 1, bistability exists among the phases. Actually, this ratio controls the Rydberg-blockade and antiblockade effect, thus the phase transition in this system can be considered as a possible approach to study both effects.
\end{abstract}

PACS numbers: 32.80.Rm 42.65.Pc 32.80.Ee

Introduction: Rydberg atoms with principal quantum number $n \gg 1$ have exaggerated atomic properties including strong dipole-dipole interactions and long radiative lifetimes [1]. These properties are attractive for quantum information processing [2, 3] and quantum many-body dynamics simulation [4, [5]. Most of these researches require a negligible spontaneous emission for reducing the quantum decoherence [6]. However, a recent research shows that when spontaneous emission is significant, quantum nonequilibrium dynamics could be demonstrated using Rydberg atom gases 7]. The system investigated is like a spin- $1 / 2$ particle system, which undergoes a phase transition from a spatially uniform phase to an antiferromagnetic phase by tuning the laser frequency as shown in Ref. [7]. Moreover, the nonequilibrium induced by the spontaneous emission leads to an oscillatory phase. Further research has found collective quantum jump between a state of low Rydberg population and a state of high Rydberg population [8]. These researches open up a new window to use Rydberg atoms for quantum nonequilibrium dynamics simulation [9].

When two atoms are close, the excitation of one atom is prohibited by an already excited neighboring atom due to the level shift by the strong dipole-dipole interaction at a short distance [10]. This phenomenon is called dipole blockade effect and has played an important role in current Rydberg atom researches [11 13]. However, recent theoretical and experimental investigations have shown that in a three-level two-photon Rydberg excitation scheme, there also exists antiblockade effect due to the Aulter-Townes splitting induced by the lower transition [14, 15]. The possibility of enhancing the antiblockade effect by trapping atoms within a lattice has been proposed [15]. So far, the exploration of physical effect of the coexistence of blockade effect and antiblockade effect on quantum dynamics of Rydberg atom gases in this two-step excitation scheme still remains at its early stage.

In this paper, we propose to study the quantum nonequilibrium dynamics of three-level atoms trapped within a lattice via a two-step excitation. Adopting the mean field approach used in Ref. 7], we predict tran-

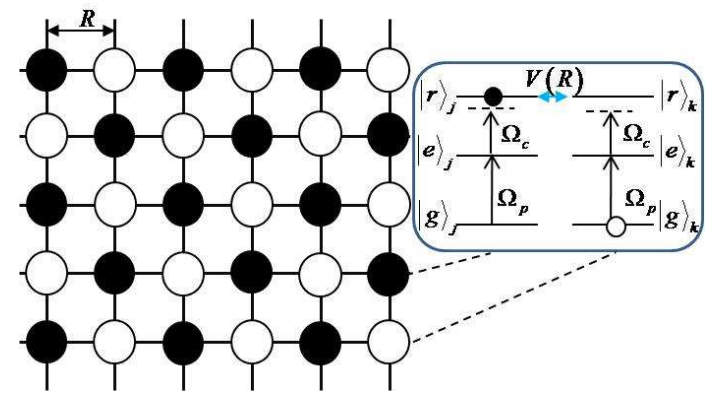

FIG. 1: Atoms trapped in an optical lattice are uniformly excited to the Rydberg state $|r\rangle$ from the ground state $|g\rangle$ via an intermediate state $|e\rangle$. The lattice is divided into two sublattices, marked respectively in white and black colors. The neighboring atoms are coupled through the Rydberg interaction $V . \Omega_{p}$ and $\Omega_{c}$ are two Rabi frequencies respectively corresponding to two optical transitions.

sition between uniform phase, antiferromagnetic phase, and oscillatory phase. Compared to the two-level system investigated in Ref. 7], we have more control knobs, including the ratio of intensity of the pump light to the control light and that of two-photon detuning to the Rydberg interaction strength, to control the phases. When the latter ratio is less than 1 and the two-photon detuning is blue-shifted, bistability exists among the phases. The latter ratio controls the competition of the blockade with antiblockade effect. Thus, both effects could be investigated through the phase transitions.

Model: Our scheme is shown in Fig. 1) Ultracold atoms are trapped within an optical lattice. We assume the lattice depth is deep enough, so that the center-ofmass motion of the atoms can be neglected. We also assume there is exactly one atom per site. All the atoms are uniformly excited by two laser beams to a Rydberg state. The laser-atom coupling scheme is shown in the right side of Fig. 1, where $|g\rangle,|e\rangle$ and $|r\rangle$ denote the ground, intermediate and Rydberg states, respectively, for the $j$-th atom, and $\Omega_{p(c)}$ is the Rabi frequency for the pump (control) laser. For convenience, we divide the lat- 
tice into two sublattices: the black sublattice and white sublattice, as shown in Fig. 1. We label the black/white site with $j / k$.

Similar configuration is used in Ref. [7], where the intermediate state $|e\rangle$ is adiabatically eliminated for a large detuning of the $|g\rangle \rightarrow|e\rangle$ transition from pump laser frequency, and an effective two-level model can be adopted [16]. In contrast, in our paper, a resonant pump laser is assumed to couple the ground state $|g\rangle$ and the intermediate state $|e\rangle$. Such a three-level model allows both the Rydberg-blockade effect and the antiblockade effect to be studied simultaneously. Whereas, in the effective two-level model, only the Rydberg-blockade was studied [12].

In the interaction picture, the Hamiltonian $H_{T}$ describing such a lattice of atoms can be given by $(\hbar=1)$ $H_{T}=\sum_{j} H_{j}+V \sum_{\langle j k\rangle}|r\rangle\left\langle\left. r\right|_{j} \otimes \mid r\right\rangle\left\langle\left. r\right|_{k}\right.$. Here, $H_{j}$ describing the interaction between the $j$-th three-level atom and two lasers is given by $H_{j}=-\delta|r\rangle\left\langle\left. r\right|_{j}+\left(\Omega_{p}|g\rangle\left\langle\left. e\right|_{j}+\right.\right.\right.$ $\Omega_{c}|e\rangle\left\langle\left. r\right|_{j}+\right.$ h.c.) with $\delta$ the two-photon detuning between states $|g\rangle_{j}$ and $|r\rangle_{j}$. $V$ is the dipole-dipole interaction between the two Rydberg atoms described by a van der Waals (vdW) potential.

The quantum dynamics for this system is governed by the master equation of its density operator $\rho$ [17]:

$$
\dot{\rho}=-i\left[H_{T}, \rho\right]+\gamma \sum_{j}\left(|g\rangle\left\langle\left. e\right|_{j} \rho \mid e\right\rangle\left\langle\left. g\right|_{j}-\frac{\left\{|e\rangle\left\langle\left. e\right|_{j}, \rho\right\}\right.}{2}\right),\right.
$$

Here, decoherence due to the spontaneous emission of the intermediate state $|e\rangle_{j}$ with its linewidth $\gamma$ is phenomenologically introduced. Dissipation from the spontaneous emission of Rydberg state $|r\rangle_{j}$ is ignored due to its relatively long lifetime $(\sim 100 \mu \mathrm{s})$ compared to $\gamma^{-1}$ [3].

Now we first investigate the steady-state solutions for Eq. (1) using the mean field approximation [18]. With this approximation, for the $j$-th atom, the Rydberg interaction $|r\rangle\left\langle\left. r\right|_{j} \otimes \sum_{k} \mid r\right\rangle\left\langle\left. r\right|_{k}\right.$ ( $k$ sums over all the nearest neighboring sites of the $j$-th site) can be replaced by a formula of density operator $|r\rangle\left\langle\left. r\right|_{j} \sum_{k} \rho_{k, r r}\right.$, thus the motional equations for the density operator elements $\rho_{j, x y}$ $(x, y=g, e, r)$ are

$$
\begin{aligned}
& \dot{\rho}_{j, r r}=2 \Omega_{c} \operatorname{Im} \rho_{j, e r} \\
& \dot{\rho}_{j, e e}=2 \Omega_{c}\left(\chi \operatorname{Im} \rho_{j, g e}-\operatorname{Im} \rho_{j, e r}\right)-\rho_{j, e e} \\
& \dot{\rho}_{j, g e}=i \Omega_{c}\left(\chi\left(1-\rho_{j, r r}-2 \rho_{j, e e}\right)+\rho_{j, g r}\right)-\frac{\rho_{j, g e}}{2} \\
& \dot{\rho}_{j, e r}=i \Omega_{c}\left(\rho_{j, e e}-\rho_{j, r r}-\chi \rho_{j, g r}\right)-i \delta_{e f f} \rho_{j, e r}-\frac{\rho_{j, e r}}{2}
\end{aligned}
$$

$$
\dot{\rho}_{j, g r}=i \Omega_{c}\left(\rho_{j, g e}-\chi \rho_{j, e r}\right)-i \delta_{e f f} \rho_{j, g r}
$$

In deducing Eqs.(2), we have scaled the frequencies and time respectively with $\gamma$ and $\gamma^{-1}$. We have also introduced the ratio of the pump Rabi frequency to the control
Rabi frequency, $\chi \equiv \Omega_{p} / \Omega_{c}$, and the effective two photon detuning $\delta_{\text {eff }} \equiv \delta-V \sum_{k} \rho_{k, r r}$. Without the RydbergRydberg interaction $V$, Eqs. (2) are the typical optical Bloch equations. The role of the Rydberg-Rydberg interaction here is to compensate the two-photon detuning $\delta$, as shown in the formula for $\delta_{e f f}$, and thus an antiblockade effect [14, 15] can be induced when $\delta_{\text {eff }} \approx 0$.

We now start to investigate the stationary solution of Eqs.(2). These stationary solutions can be put into three classes:

(a) Uniform phase (uni): The atoms are uniformly excited over the whole lattice, i.e., $\rho_{j, r r}^{s}=\rho_{k, r r}^{s}$. Hereafter, the superscript $s$ stands for the stationary solution.

(b) Antiferromagnetic phase (AF): One sublattice has higher excitation than the other, i.e., $\rho_{j, r r} \neq \rho_{k, r r}$.

(c) Oscillatory phase (osc): Rydberg population oscillates periodically in time between two sublattices. In this case, one cannot find any stable steady-state AF solutions.

We first study the uniform phase $\left(\rho_{j(k), x y}^{s}=\rho_{x y}^{s}\right)$. From the stationary solution of Eqs.(2), we obtain a cubic equation for $\rho_{x y}^{s}$ :

$$
\begin{aligned}
& \left(\rho_{r r}^{s}\right)^{3}-\frac{2 \delta}{V}\left(\rho_{r r}^{s}\right)^{2}+\frac{1}{V^{2}}\left(\delta^{2}+\frac{4 \Omega_{c}^{4}\left(\chi^{2}+1\right)^{2}}{1+8 \chi^{2} \Omega_{c}^{2}}\right) \rho_{r r}^{s} \\
& -\frac{4 \Omega_{c}^{4} \chi^{2}\left(\chi^{2}+1\right)}{V^{2}\left(1+8 \chi^{2} \Omega_{c}^{2}\right)}=0
\end{aligned}
$$

For AF phase, two sublattices are Rydberg-populated differently, and labeled 1 and 2 in the subscript of $\rho_{j(k), x y}^{s}$. From Eqs.(2), we obtain equations involving $\rho_{1, r r}^{s}$ and $\rho_{2, r r}^{S}$ :

$$
\begin{aligned}
& \rho_{1, r r}^{s}+\frac{\left(1+8 \Omega_{c}^{2} \chi^{2}\right)\left(V \rho_{2, r r}^{s}-\delta\right)^{2}}{4 \Omega_{c}^{4}\left(1+\chi^{2}\right)^{2}} \rho_{1, r r}^{s}=\frac{\chi^{2}}{1+\chi^{2}}, \\
& \rho_{2, r r}^{s}+\frac{\left(1+8 \Omega_{c}^{2} \chi^{2}\right)\left(V \rho_{1, r r}^{s}-\delta\right)^{2}}{4 \Omega_{c}^{4}\left(1+\chi^{2}\right)^{2}} \rho_{2, r r}^{s}=\frac{\chi^{2}}{1+\chi^{2}} .
\end{aligned}
$$

The solutions $\rho_{1(2), r r}^{s}$ of the coupled equations (4) and (5) correspond to two roots of the following equation

$$
\left(\rho_{r r}^{s}\right)^{2}-2 A \rho_{r r}^{s}+B=0,
$$

where $A=\delta / V+2 \Omega_{c}^{4} \chi^{2}\left(1+\chi^{2}\right) /\left[4 \Omega_{c}^{4}\left(1+\chi^{2}\right)^{2}+\delta^{2}\left(1+8 \chi^{2} \Omega_{c}^{2}\right)\right]$ $B=\delta^{2} / V^{2}+4 \Omega_{c}^{4}\left(1+\chi^{2}\right)^{2} /\left[V^{2}\left(1+8 \chi^{2} \Omega_{c}^{2}\right)\right]$.

When $A^{2}-B<0$, the quadratic equation (6) has complex roots. In this situation, the $\mathrm{AF}$ and oscillatory phases don't exist, and only uniform phase exists. In other cases, the quadratic equation (6) has one pair of real solutions. When the real solutions are stable against small perturbation, the system is in the AF phase; otherwise, the system is in oscillatory phase.

Phase Diagram: In this section, we show the numerical results for the stationary solution with the experimentally tunable two-photon detuning $\delta$ for $\chi=5$ (Fig. 

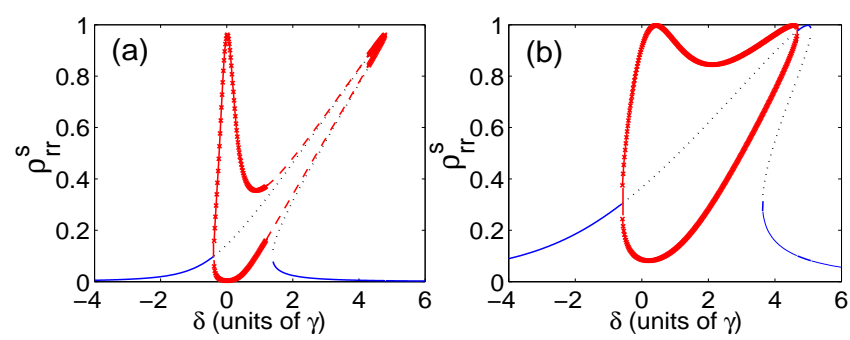

FIG. 2: (color online) Stationary solutions $\rho_{r r}^{s}$ as a function of detuning $\delta$ with $V=5, \Omega_{c}=0.1$ for different ratios (a) $\chi=5$ and (b) $\chi=20$. The stable and unstable uniform phases respectively show in solid line and dotted line. AF phase and oscillatory phase are repsectively shown in solid line with cross symbols, and dashed line.

26) and 20 (Fig. 2b). Stable and unstable uniform phases governed by Eq. (3) are respectively plotted in solid lines and dotted lines. AF phases and oscillatory phases from Eq. (6) are respectively plotted in solid lines with cross symbols and dashed lines.

In Fig. 2(a), there exists only one stable uniform phase for small detunings $\delta \leq \delta_{c} \approx-0.5$. The critical point $\delta_{c}$ can be determined by $A^{2}=B$. When $\delta$ crosses the critical point, the uniform phase becomes unstable, giving rise to a pair of AF phases. This is the pitchfork bifurcation of supercritical type [19], in which the uniform fixed point loses its stability and two stable AF fixed points appear. As $\delta$ increases further to the value larger than 1.22 , the AF phase becomes unstable and the system enters into the oscillatory phase.

The phases can be controlled experimentally by adjusting the ratio of the pump Rabi frequency to the control one, $\chi$. With a very strong pump transition $\left(\Omega_{p} \gg \Omega_{c}\right)$, the Rydberg-excitation rate can not catch up with the decay rate from intermediate state, so that population in Rydberg state hardly changes. In Fig. 2(b) with $\chi=20$ much larger than that in Fig. 2(a), the stable AF fixed points always exist and there is no oscillatory phase. There is a supercritical pitchfork bifurcation at $\delta=4.67$ where the uniform phase becomes stable again.

Figures 2(a) and (b) also show the signals of Rydbergblockade effect and antiblockade effect. When $\delta=0$, $\left|\rho_{1, r r}^{s}-\rho_{2, r r}^{s}\right| \approx 1$ for AF phase. This means a resonant two-photon detuning leads to an entire Rydberg excitation in one sublattice, whereas the other sublattice is strongly blocked. When $\delta$ increases to $5.0, \rho_{1, r r}^{s}=$ $\rho_{2, r r}^{s} \approx 1.0$, and the two-photon detuning $\delta$ is compensated by the Rydberg energy shift $\left(\delta_{\text {eff }} \approx 0\right)$, indicating the antiblockade effect.

Since the stationary solutions are dependent on both the two-photon detuning $\delta$ and the ratio of the Rabi frequencies for pump and control lasers $\chi$, we plot the phase diagram in the parameter space of $(\delta, \chi)$ in Fig. 3. The number of stable uniform phases is denoted in a bracket. The position that $\delta_{\text {eff }}=0$ has been marked. When $\delta_{\text {eff }} \gtrsim 0\left(\delta \gtrsim V \sum_{k} \rho_{k, r r}\right)$, the Rydberg interaction plays

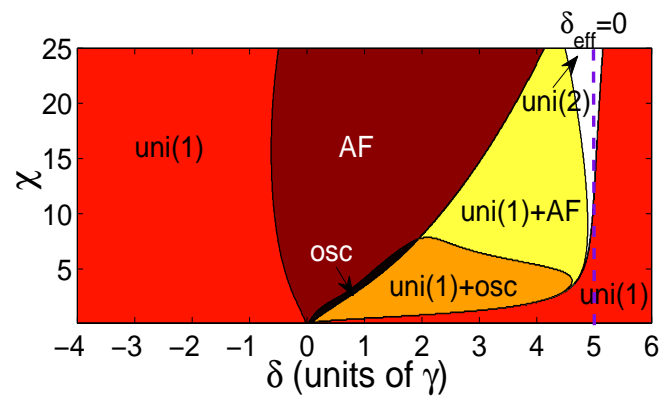

FIG. 3: (color online)Phase diagram within $(\delta, \chi)$ space for $V=5, \Omega_{c}=0.1$.

negligible role, and thus the system behaves like a noninteracting particle system. Consequently, there is only one stable uniform phase, no matter how $\chi$ varies. When $\delta$ is comparable to $V \sum_{k} \rho_{k, r r}$, there is complex phase distribution within $(\delta, \chi)$ space. $\mathrm{AF}$ phase contains a pair of stable nonuniform fixed points. In the regions marked with "uni(2)", "uni(1)+AF" and "uni(1)+osc", bistability takes place between two uniform phases, or uniform and antiferromagnetic phase or uniform and oscillatory phase. When the two-photon detuning $\delta$ is resonant $(\delta=0)$, the system is in AF phase. When $\delta$ is red shifted $(\delta<0)$ and near-resonant $(\delta \approx 0)$, there could be $\mathrm{AF}$ phase or uniform phase depending on the ratio $\chi$; however, if $\delta$ is further red-shifted, there is only uniform phase.

Rydberg-Rydberg interaction: The phase diagram analysis has shown that the Rydberg-Rydberg interaction strength could have strong influence on the dynamics of Rydberg gases. In experiments, the Rydberg-Rydberg interaction strength can be controlled with the atomatom separation $R$. Now we take an isotropic vdW interaction $V=-d C_{6} / \gamma R^{6}$ ( $d$ is the lattice dimension) for short separation $R$ as an example to investigate the controlling of the Rydberg gas dynamics with the lattice size 20,21$]$. For ${ }^{87} \mathrm{Rb}$ atoms, we choose $5 s, 5 p$ and Rydberg state respectively as $|g\rangle_{j},|e\rangle_{j}$ and $|r\rangle_{j}$. The interaction coefficient for Rydberg atoms is $C_{6}=-870 \mathrm{kHz} \mu \mathrm{m}^{6}$ when $|r\rangle_{j}=23 s_{1 / 2}$ [22], and linewidth $\gamma$ from state $|e\rangle_{j}$ is $\gamma / 2 \pi=1.7 \mathrm{MHz}$ [23]. Using these parameters, the normalized vdW potential is $V \approx 1 / R^{6}$ ( $R$ is in units of $\mu \mathrm{m}, d=2)$. In this case, we obtain stationary solutions $\rho_{r r}^{s}$ with $\delta=0$ as a function of spacing $R$ as shown in Fig. 团 for $\chi=5$ (dashed lines) and 20 (solid lines).

When $R \ll 1.0 \mu \mathrm{m}$, the system is dominated by a pair of AF phase with one sublattice completely Rydbergexcited $\left(\rho_{r r}^{s} \approx 1\right)$ and the other blockade $\left(\rho_{r r}^{s} \approx 0\right)$. This is a demonstration of the Rydberg blockade phenomenon under strong Rydberg interaction [24]. From experimental view, $R \ll 1.0 \mu \mathrm{m}$ can be realized in solid systems, but it is hard for atoms trapped within a conventional optical lattice [25]. However, subwavelength optical lattices can be facilitated with a recent progress in subwavelength focusing, and have been realized recently for 


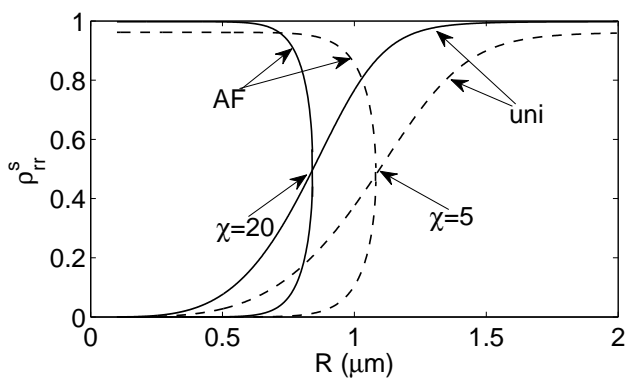

FIG. 4: (color online)Stationary solutions $\rho_{r r}^{s}$ as a function of lattice spacing $R$ for $\chi=5$ (dashed lines) and $\chi=20$ (solid lines).
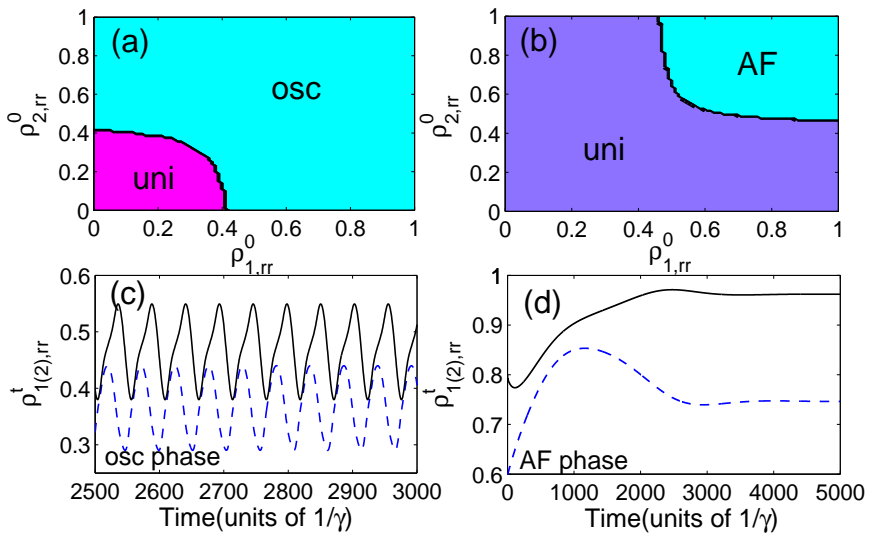

FIG. 5: (color online) Phase diagram in the space of initial conditions $\left(\rho_{1, r r}^{0}, \rho_{2, r r}^{0}\right)$ with (a) $\chi=5, \delta=2$ and (b) $\chi=20$, $\delta=4$. (c) and (d) show typical Rydberg population dynamics of oscillatory phase $\left(\rho_{1, r r}^{0}=0.4, \rho_{2, r r}^{0}=0.6\right)$ and $\mathrm{AF}$ phase $\left(\rho_{1, r r}^{0}=0.6, \rho_{2, r r}^{0}=0.8\right)$, respectively.

matter wave Bloch oscillation [26]. Thus, observing AF phase is promising in lattices created with subwavelength focusing techniques.

When $R$ increases, the uniform phase develops and one sees the two-phase (uni and AF) coexistence in the regime around $R \sim 1 \mu \mathrm{m}$. After crossing over this regime, the AF phase disappears and the uniform phase dominates with $R$ increasing further, due to the negligible value of $V$ for large atom-atom separations.

Dynamics: The dynamical evolution of the system is explored via a straightforward time-integration of the mean-field equations (2). According to the phase diagram of Fig. 3, the system is in the bistable state between the uniform phase and oscillatory phase for $\chi=5, \delta=2$. The results of dynamical simulation in Fig. 55(a) gives the information about which phase the system will finally evolve into by choosing different initial Rydberg populations $\left(\rho_{1, r r}^{0}, \rho_{2, r r}^{0}\right)$. If both $\rho_{1, r r}^{0}$ and $\rho_{2, r r}^{0}$ are smaller than 0.4 , the final state remains in the uniform phase. By increasing $\rho_{1, r r}^{0}$ or $\rho_{2, r r}^{0}$, due to the bistability effect, the system enters into oscillatory phase with Rydberg population periodically oscillating between two sublattices(see (c) for $\rho_{1, r r}^{0}=0.4, \rho_{2, r r}^{0}=0.6$ ). Fig. 5(b) is obtained in the same way except with $\chi=20, \delta=4$. Under this set of parameters, the system is in the bistable state between uniform phase and AF phase according to Fig. 3. The uniform phase now becomes dominated. This is because with a far-off resonant two-photon detuning and a weak control laser, the transition from intermediate state to Rydberg state becomes very difficult, leading to small Rydberg population and in turn weak Rydberg interaction. Therefore the system tends to stay in the uniform phase. However, as long as $\rho_{1, r r}^{0}$ and $\rho_{2, r r}^{0}$ are both significantly large, the system will still evolve into the AF phase. Fig. 5(d) presents a typical Rydberg population dynamics in the case of AF phase for $\rho_{1, r r}^{0}=0.6$, $\rho_{2, r r}^{0}=0.8$.

Conclusions: We have studied mean-field phase diagrams in a lattice system of three-level atoms with twophoton Rydberg excitation and spontaneous emissioninduced dissipation. We have shown that the system has rich phases, including antiferromagnetic phase, uniform phase, and oscillatory phase. We can control the phases using either the ratio of the intensity of the pump to the control light, or that of two-photon detuning to the Rydberg interaction strength. The latter ratio actually tunes the competition of the Rydberg-blockade effect and the antiblockade effect. We found when the Rydberg interaction strength $V$ is much larger than two-photon detuning $\delta$ (the Rydberg-blockade dominates), the system can be in the AF phase. When $V$ is comparable to $\delta$, the competition of the Rydberg-blockade eand the antiblockade effects leads to bistability of the system. When $|\delta|$ is far larger than $V$, the system is in the uniform phase. Our work shows the possibility of studying antiblockade effect within a lattice [15].

We thank T. Lee for useful discussions. This work was supported by National Basic Research Program of China (973 Program) under Grant No. 2011CB921604, 2011CB921602, the NSFC under Grant Nos. 11104076, 11004057, 11034002, 10974057 and 10874045, the Specialized Research Fund for the Doctoral Program of Higher Education No. 20110076120004, the "Chen Guang" project supported by Shanghai Municipal Education Commission and Shanghai Education Development Foundation under Grant No. 10CG24
[1] T. F. Gallagher, Rydberg Atoms (Cambridge University Press, Cambridge, 1994)..

[2] M. D. Lukin et al., Phys. Rev. Letts. 87037901 (2001).
[3] M. Saffman, T. G. Walker and K. MØlmer, Rev. Mod. Phys. 822313 (2010) and references therein.

[4] I. Buluta and F. Nori, Science 326108 (2009). 
[5] H. Weimer et al., Nature Phys. 6382 (2010).

[6] G. Pupillo et al., Phys. Rev. Letts. 104223002 (2010).

[7] T. E. Lee, H. Häffner and M. C. Cross, Phys. Rev. A 84 031402 (2011); I. Lesanovsky Physics 471 (2011).

[8] T. E. Lee, H. Häffner and M. C. Cross, Phys. Rev. Letts. 108023602 (2012).

[9] S. Diehl et al., Nature Phys. 4878 (2008).

[10] M Viteau et al., Phys. Rev. Letts. 107060402 (2011).

[11] T. F. Gallagher and P. Pillet, Advances in Atomic, Molecular, and Optical Physics 56161 (2008).

[12] E. Urban et al., Nature Phys. 5110 (2009); M. Weidemüller Nature Phys. 591 (2009).

[13] D. Comparat and P. Pillet, J. Opt. Soc. Am. B 27 A208 (2010), and references therein.

[14] C. Ates, T. Pohl, T. Pattard and J. M. Rost, Phys. Rev. Letts. 98023002 (2007).

[15] T. Amthor, C. Giese, C. S. Hofmann and M. Weidemüler, Phys. Rev. Letts. 104 013001(2010).

[16] Heping Zeng, Weiping Zhang and Fucheng Lin, Phys.
Rev. A 522155 (1995).

[17] C. W. Gardiner and P. Zoller, Quantum Noise (SpringerVerlag 2004).

[18] P. M. Chaikin and T. C. Lubensky Principles of condensed matter physics (Cambridge University Press, 2007).

[19] S. N. Rasband, Chaotic Dynamics of Nonlinear Systems (New York: Wiley, 1990)..

[20] O. N. Osychenko et al., Phys. Rev. A 84063621 (2011).

[21] S. Ji, C. Ates, and I. Lesanovsky, Phys. Rev. Letts. 107 060406 (2011).

[22] A. Reinhard, T. C. Liebisch, B. Knuffman and G. Raithel, Phys. Rev. A 75032712 (2007).

[23] J. D. Pritchard et al., Phys. Rev. Letts. 105193603 (2010).

[24] A. Gaëtan et.al. Nat. Phys. 5115 (2009).

[25] H. Kübler et al., Nature Photon. 4 112(2010).

[26] T. Salger et al., Phys. Rev. A 79 011605(R) (2009). 